\title{
Oncoplastic technique using a lateral mammary adipofascial flap for the breast-conserving reconstruction
}

\author{
Jiang Wu, Changjiao Yan, Lei Wang, Yuqing Yang, Rui Ling, Nanlin Li \\ Department of Thyroid, Breast, and Vascular Surgery, Xijing Hospital, Fourth Military Medical University, Xi'an 710032, China \\ Correspondence to: Nanlin Li; Rui Ling. Department of Thyroid, Breast, and Vascular Surgery, Xijing Hospital, Fourth Military Medical University, \\ Xi'an 710032, China. Email: linanlingo@126.com; lingruiaoxue@126.com.
}

\begin{abstract}
Achieving satisfactory cosmetic results after breast-conserving surgery (BCS) can be difficult for breast cancer patients with large tumors. To avoid breast asymmetries and deformation, we present a case of breast reconstruction after BCS with a lateral mammary adipofascial flap, which was dissociated along the sentinel lymph node biopsy incision. During the procedure, the pedicled flap was transferred into the residual tumor cavity through a tunnel between them and then matched the residual cavity. The sufficient blood supply of the transferred flap was observed in ultrasound at follow-up. The operative is straightforward to perform and less invasive. No operative complications were observed. The cosmetic results were found to be good with a natural shape, volume, and symmetry, and with which the patient has reported high satisfaction. This procedure offers a new possibility in breast reconstruction and is worth considering as a new option for immediate repair after BCS.
\end{abstract}

Keywords: Breast-conserving surgery (BCS); autologous tissue; adipofascial flap

Submitted Nov 14, 2019. Accepted for publication Nov 27, 2019.

doi: $10.21037 /$ atm.2019.12.12

View this article at: http://dx.doi.org/10.21037/atm.2019.12.12

\section{Introduction}

Breast-conserving surgery (BCS) has become a standard operative strategy for invasive breast cancer $(1,2)$. The purpose of BCS is to acquire local control and provide as close as possible to the normal look and feel of a natural breast (3). However, achieving satisfactory cosmetic results can be difficult for patients with the extent of the tumor is resected, that is large. Immediate reconstruction using autologous tissue has been popularized to avoid breast asymmetries and deformation after BCS $(4,5)$. The following is a case report of a patient who presented for tissue flap transfer residual cavity repair surgery after a BCS with lateral mammary adipofascial flap. This design can reduce surgical trauma, shorten the operation time, and offer a new possibility in the setting of earlier autologous breast reconstruction.

\section{Case presentation}

A 48-year-old female patient was admitted to our department for further examination after noticing a lump in her right breast. A tumor, $2.0 \mathrm{~cm}$ from the nipple, was palpated in the outer lower quadrant of the right breast with a diameter of $2.0 \mathrm{~cm}$, without dimple sign or orange peel sign. Ultrasonography revealed a $2.1 \times 1.8 \times 1.3 \mathrm{~cm}^{3}$ irregularly shaped low-echoic mass in the right outer lower quadrant (seven o'clock direction), and no enlarged lymph nodes in both axillae. Mammography revealed no mass or calcification in the left breast, but an irregularly shaped mass and a diffusely distributed fine calcification were detected in the right breast (Figure 1). MRI showed an irregular shaped mass in the right outer lower quadrant, which was burr-like and extended to the nipple and lateral. Core needle aspiration cytology of the lesion confirmed 
that the tumor was invasive ductal carcinoma. The tumor was positive for the estrogen and progesterone receptors and human epidermal growth factor receptor 2 (HER2) on immunohistochemical examination. No distant metastases were apparent. The extent of the tumor being resected would be large due to the tumor was close to the nipple and was "crab-legged" outward. Therefore, conventional BCS may result in breast asymmetry or deformity. However, the patient who is a dance teacher had an ardent desire to conserve the breast. Therefore, we proposed "tissue flap transfer residual cavity repair surgery" after BCS, which obtained the patient's approval.

The surgical flow chart of the patient, as shown in Figure 2 and the surgical schematic diagram was shown in Figure 3. With the patient in a supine position, the incision of BCS and the edge of the tissue flap for transfer were

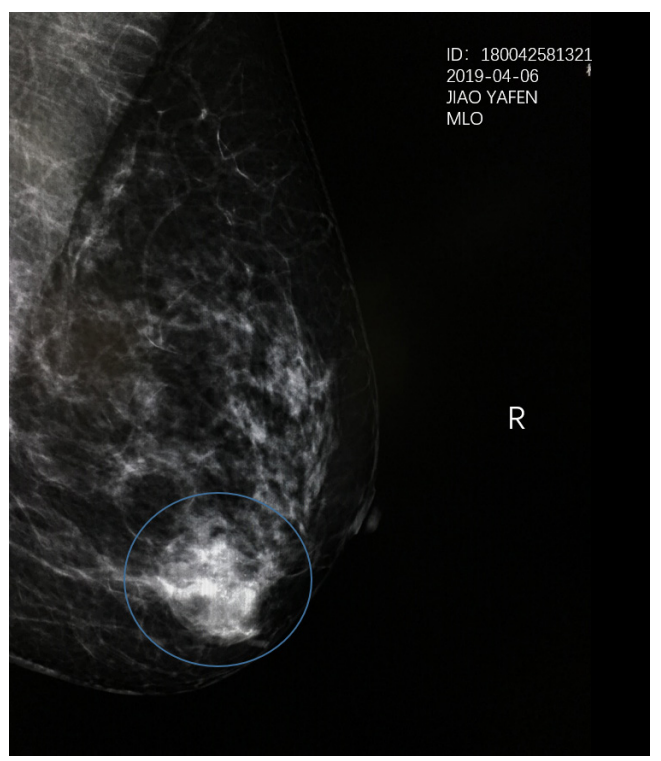

Figure 1 Mammography of the patient's right breast. The part of the blue circle showed an irregularly shaped mass and a diffusely distributed fine calcification closed to the nipple. marked on the skin surface (Figure $4 A$ ). After removing four methylene blue-stained sentinel lymph nodes (Figure 4B,C), tumor resection was conducted with a $10 \mathrm{~mm}$ macroscopic margin in every direction (Figures $3 A, B, 4 D$ ). Then intraoperative frozen pathological diagnosis confirmed the four sentinel lymph nodes and margins in BCS (Figure 4E) were negative. After lengthening about $4 \mathrm{~cm}$ longitudinally along the axillary incision, the pedicled tissue flap that preserved lateral thoracic vessels was dissociated (Figures $3 C, 4 F$ ). During the procedure, the flap was dissected to the breast gland and reshaped to match the residual cavity of the tumor. Then the subcutaneous tissues were separated to form a tunnel to the residual cavity (Figure $4 G$ ). The color and blood supply of the flap were observed. Next, the flap was transferred into the residual cavity through the tunnel and sutured with the breast gland (Figures 3D,E,4H). Finally, following clean and disinfection of the incisions (Figure 4I), the incisions closed (Figures 3F,4f).

As shown in Figure 5, the postoperative blood supply was evaluated by color-flow ultrasound imaging. The blood supply of the transferred flap was observed in ultrasound after two weeks and two months after the operation. The postoperative finding is shown in Figure 6. She was satisfied with her breasts because the natural feel and symmetry were obtained, and no partial flap loss, seroma, or flapsite infection was developed. Postoperatively, the patient would receive four rounds of combined chemotherapy with epirubicin (E), cyclophosphamide (C), then docetaxel (T). Furthermore, she would receive Herceptin for one year and endocrine therapy for at least five years.

\section{Discussion}

When BCS is used to remove tumors that are localized to the lower quadrant or large, the breast may be asymmetric and with the displacement of the nipple. Immediate reconstruction using autologous tissue after BCS has

\begin{tabular}{|c|c|c|c|c|}
\hline $\begin{array}{l}\text { Sentinel lymph } \\
\text { node biopsy }\end{array}$ & $\begin{array}{l}\text { Tumor } \\
\text { resection }\end{array}$ & $\begin{array}{l}\text { Intraoperative frozen pathological diagnosis } \\
\text { of margins in BCS and sentinel lymph node }\end{array}$ & $\begin{array}{l}\text { Tissue flap transfer residual } \\
\text { cavity repair surgery }\end{array}$ & $\begin{array}{l}\text { Suture and bandage } \\
\text { of wounds }\end{array}$ \\
\hline
\end{tabular}

Figure 2 Surgical flow chart of the patient. 

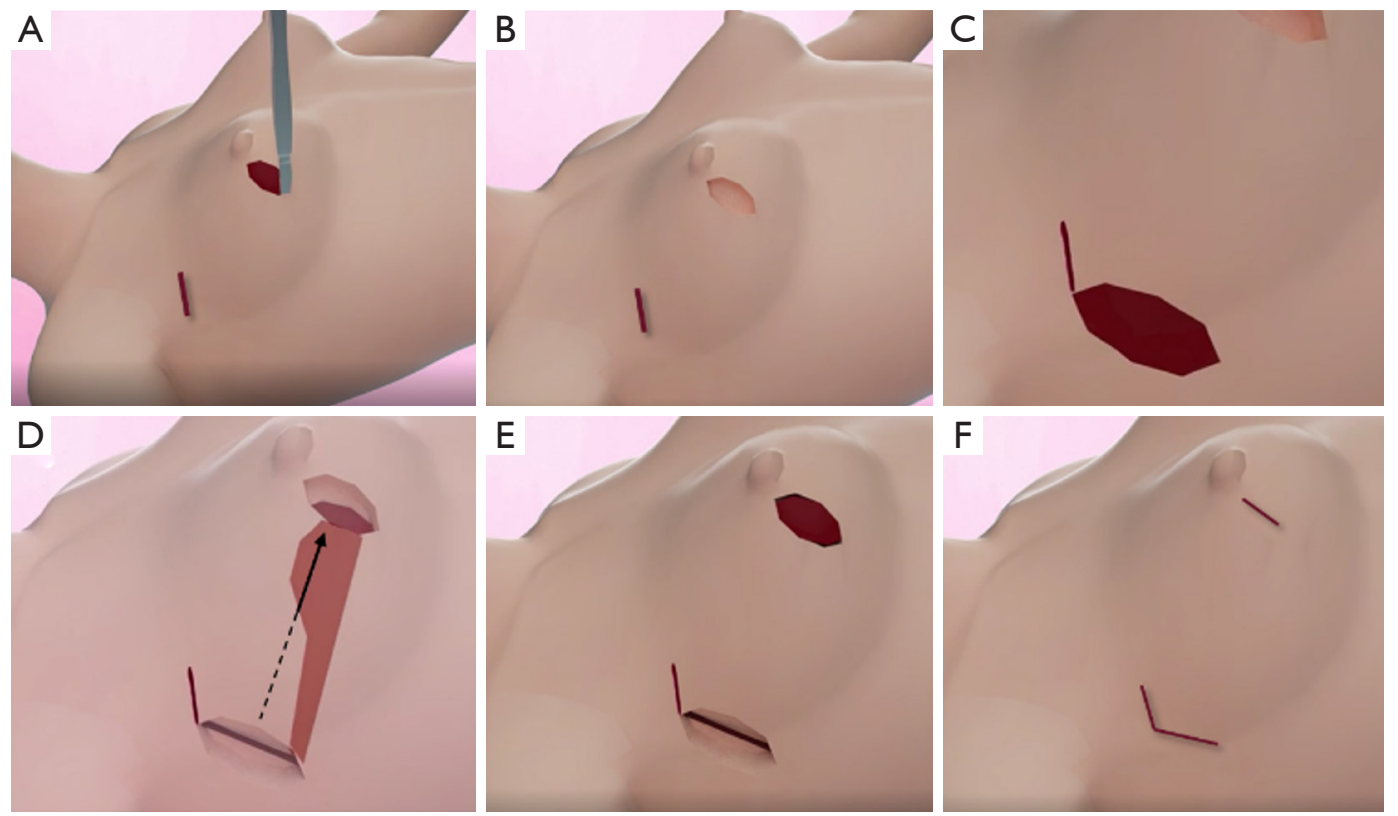

Figure 3 Surgical schematic diagram of the patient. (A) Tumor resection (the dark red part of breast represents the extent of the resection; the red line near the armpit represents the sentinel lymph node biopsy incision); (B) the residual cavity of the tumor; (C) dissociation of the pedicled tissue flap (the dark red part along the axillary incision); (D) transfer of the flap into the residual cavity through the tunnel; (E) filling the flap into the residual cavity; (F) closing incisions.
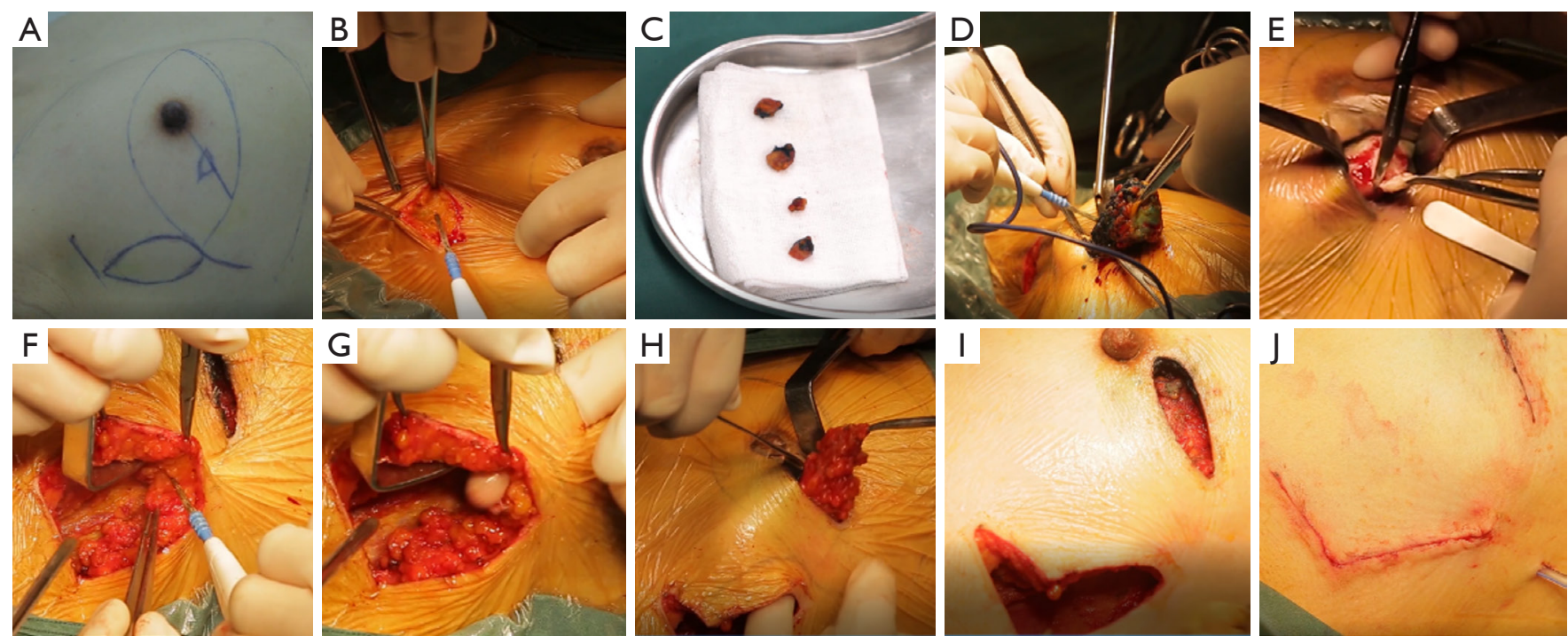

Figure 4 Photos of the surgical procedure. (A) Marking the incision of breast-conserving surgery and the edge of the tissue flap for transfer on the skin surface; (B) sentinel lymph node biopsy; (C) four methylene blue-stained sentinel lymph nodes; (D) tumor resection; (E) taking the margins in BCS; (F) dissociation of the pedicled tissue flap; $(\mathrm{G})$ forming a tunnel from the flap to the residual cavity; $(\mathrm{H})$ transfer of the flap into the residual cavity through the tunnel; (I) clean and disinfection of the incisions; (J) closing incisions. 

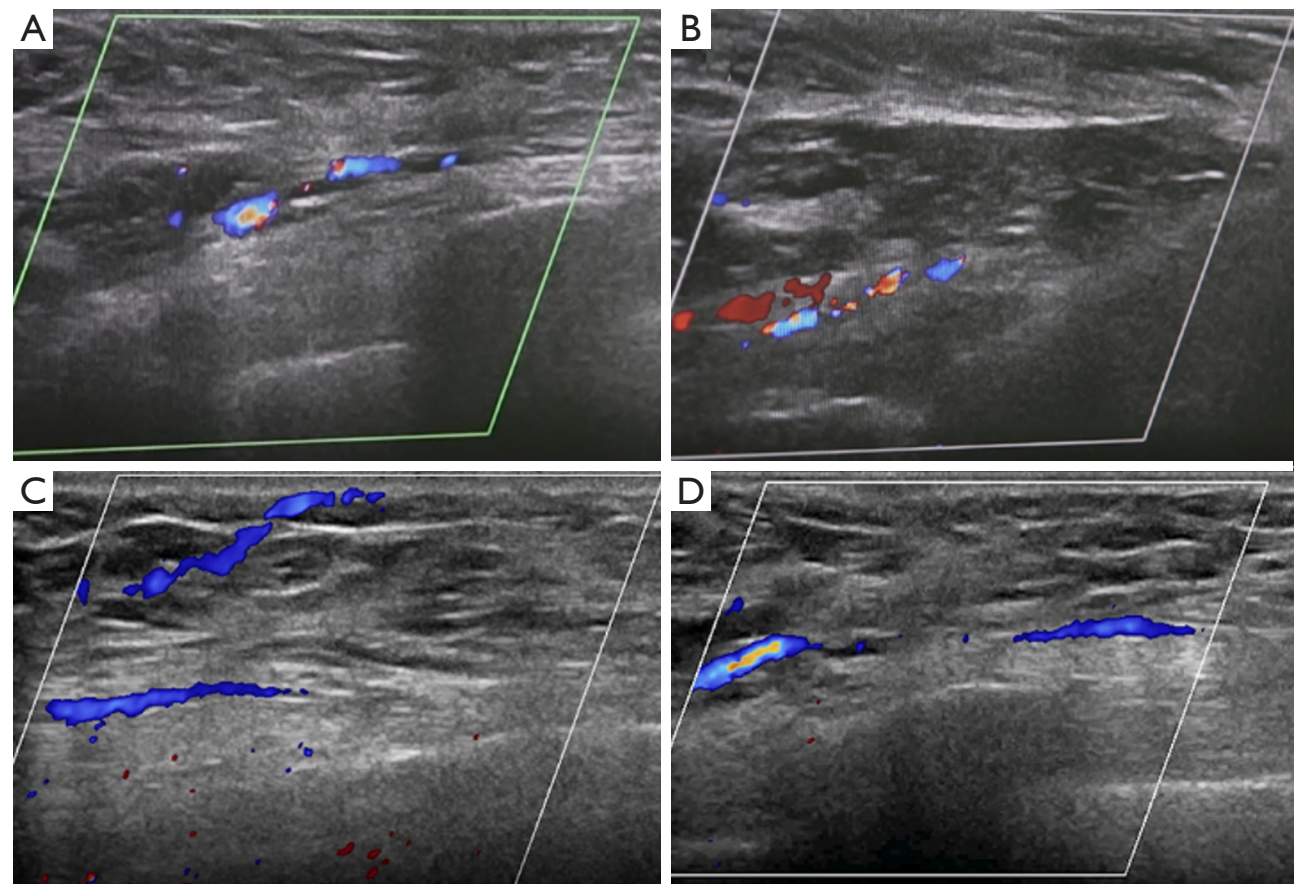

Figure 5 Color-flow ultrasound imaging to evaluate postoperative blood supply. (A,B) Two weeks after operation; (C,D) two months after the operation.
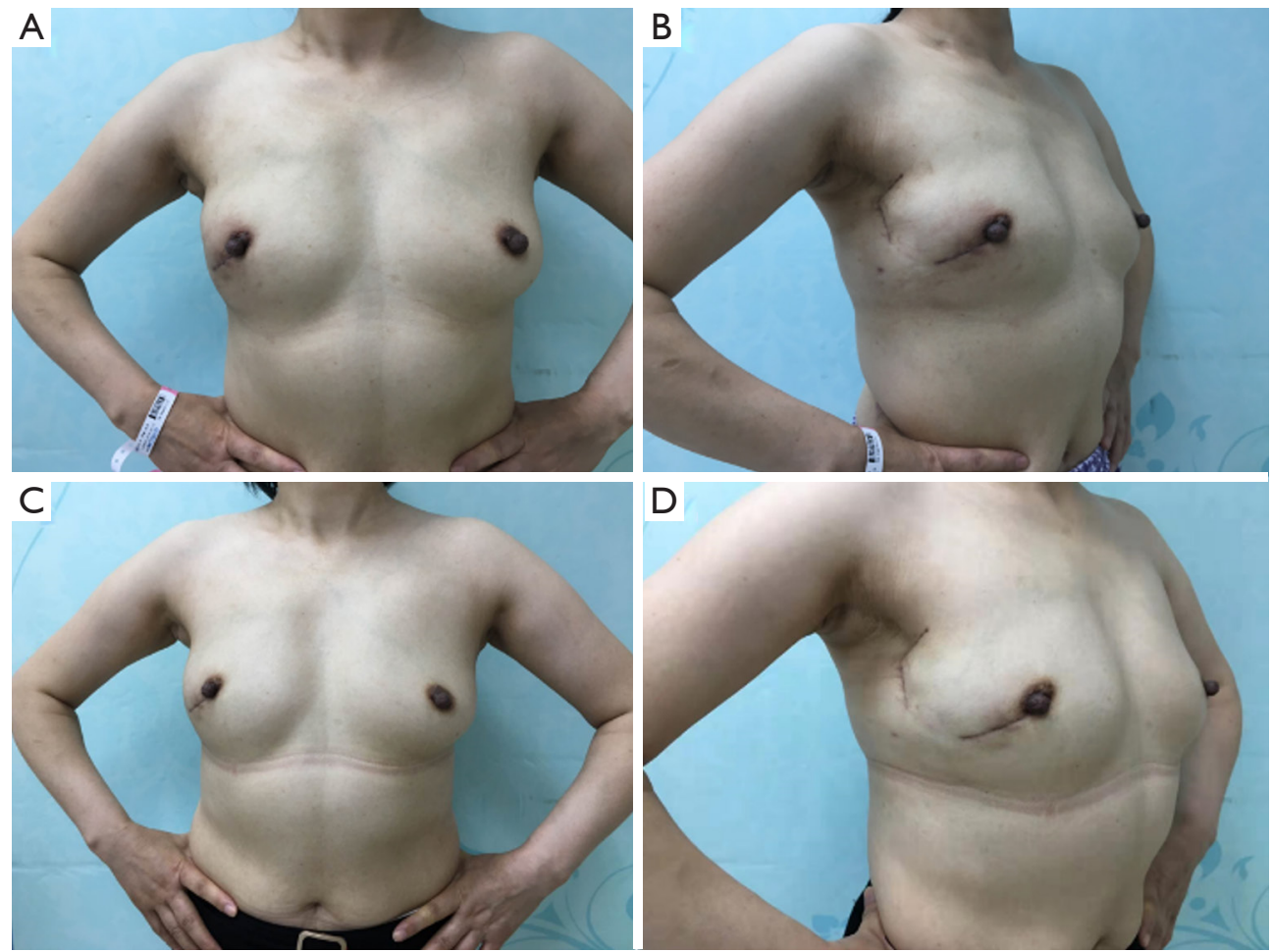

Figure 6 Postoperative photographs of the patient. (A,B) Two weeks after operation; (C,D) two months after the operation. 
been performed increasingly to avoid breast deformities. The most commonly utilized donor sites for autologous reconstruction are from the abdomen (6). They include the transverse rectus abdominis musculocutaneous (TRAM), deep inferior epigastric perforator (DIEP), and superficial inferior epigastric artery flaps (SIEA) (7). The secondary donor sites are typically considered that include the posterior thorax, gluteal, and thigh regions when the abdomen is not suitable $(8,9)$. However, these donor flaps are designed from remote areas to the breast, which results in patients having to physically tolerate several hours in length of the operation and a long recovery period following these procedures. Besides, a prerequisite for using the abdomen is that there are no scars in the critical areas, and the patient has no plan for pregnancy (10). And the abdominal flaps may be prone to weakness, and a bulge or hernia may occur (11). Latissimus dorsi flaps may be prone to seroma formation (12).

In this case, a flap that is located very close to the breast was transferred to the residual cavity after BCS, and this procedure is easy to perform. Thus, breast surgeons can perform this modality without the help of plastic surgeons. The donor site incision is small, which can be obtained by extending along the incision of the sentinel lymph node biopsy. Compared to lateral mammary adipofascial flap, in this case, TRAM, DIEP, or latissimus dorsi flaps are more invasive at the donor site. Furthermore, the transfer of lateral mammary adipofascial flap does not require microvascular techniques. Therefore, this is not only a more straightforward technique than the current conventional flaps but also a technique with short operation time and less postoperative pain to patients.

As shown in this case, the cosmetic results were found to be good with a natural shape, volume, and symmetry. The sufficient blood supply of the transferred flap was observed in ultrasound. The patient was satisfied with these results. It should be noted that the tailored flap was $4 \times 6 \mathrm{~cm}^{2}$ in this case. Because of the limited amount of tissue, this procedure is suitable for small or medium breasts. Chinese women's breasts do not tend to be large, so that this technique may offer a new choice for breast cancer patients in China and patients with small or medium breasts in other countries.

\section{Conclusions}

In conclusion, the lateral mammary adipofascial flap procedure is considered to be easy to perform with a relatively low level of invasiveness, and the cosmetic outcomes were generally good, without any complications. As a result, this procedure may be worth considering as an option for immediate repair after BCS in cases where the patient desires a less invasive operation.

\section{Acknowledgments}

None.

\section{Footnote}

Conflicts of Interest: The authors have no conflicts of interest to declare.

Ethical Statement: The authors are accountable for all aspects of the work in ensuring that questions related to the accuracy or integrity of any part of the work are appropriately investigated and resolved. Written informed consent was obtained from the patient for publication of this manuscript and any accompanying images.

\section{References}

1. van Dongen JA, Voogd AC, Fentiman IS, et al. Long-term results of a randomized trial comparing breast-conserving therapy with mastectomy: European Organization for Research and Treatment of Cancer 10801 trial. J Natl Cancer Inst 2000;92:1143-50.

2. Speers C, Pierce LJ. Postoperative Radiotherapy After Breast-Conserving Surgery for Early-Stage Breast Cancer: A Review. JAMA Oncol 2016;2:1075-82.

3. Recio-Saucedo A, Gerty S, Foster C, et al. Information requirements of young women with breast cancer treated with mastectomy or breast conserving surgery: A systematic review. Breast 2016;25:1-13.

4. Macadam SA, Bovill ES, Buchel EW, et al. EvidenceBased Medicine: Autologous Breast Reconstruction. Plast Reconstr Surg 2017;139:204e-29e.

5. Juhl AA, Redsted S, Engberg Damsgaard T. Autologous fat grafting after breast conserving surgery: Breast imaging changes and patient-reported outcome. J Plast Reconstr Aesthet Surg 2018;71:1570-6.

6. Thiessen FEF, Tjalma WAA, Tondu T. Breast reconstruction after breast conservation therapy for breast cancer. Eur J Obstet Gynecol Reprod Biol 2018;230:233-8.

7. Nahabedian MY, Patel K. Autologous flap breast reconstruction: Surgical algorithm and patient selection. J Surg Oncol 2016;113:865-74. 
8. Chopan M, White JA, Sayadi LR, et al. Autogenous Fat Grafting to the Breast and Gluteal Regions: Safety Profile Including Risks and Complications. Plast Reconstr Surg 2019; 143:1625-32.

9. Satake T, Muto M, Ko S, et al. Breast reconstruction using free posterior medial thigh perforator flaps: intraoperative anatomical study and clinical results. Plast Reconstr Surg 2014;134:880-91.

10. Rozen WM, Garcia-Tutor E, Alonso-Burgos A, et al. The effect of anterior abdominal wall scars on the vascular

Cite this article as: Wu J, Yan C, Wang L, Yang Y, Ling R, Li N. Oncoplastic technique using a lateral mammary adipofascial flap for the breast-conserving reconstruction. Ann Transl Med 2019;7(23):794. doi: 10.21037/atm.2019.12.12 anatomy of the abdominal wall: A cadaveric and clinical study with clinical implications. Clin Anat 2009;22:815-22.

11. Duraes EF, Schwarz G, Durand P, et al. Complications Following Abdominal-Based Free Flap Breast Reconstruction: Is a 30 days Complication Rate Representative? Aesthetic Plast Surg 2015;39:694-9.

12. Thorarinsson A, Frojd V, Kolby L, et al. A retrospective review of the incidence of various complications in different delayed breast reconstruction methods. J Plast Surg Hand Surg 2016;50:25-34. 